\title{
DETECTION OF CORONARY ARTERY DISEASE BY REDUCED FEATURES AND EXTREME LEARNING MACHINE
}

\author{
RAM SEWAK SINGH, BARJINDER SINGH SAINI, \\ RAMESH KUMAR SUNKARIA
}

\author{
Department of Electronics and Communication Engineering, Dr. B.R. Ambedkar \\ National Institute of Technology, Jalandhar, India
}

\begin{abstract}
Objective. Cardiovascular diseases generate the highest mortality in the globe population, mainly due to coronary artery disease (CAD) like arrhythmia, myocardial infarction and heart failure. Therefore, an early identification of CAD and diagnosis is essential. For this, we have proposed a new approach to detect the CAD patients using heart rate variability (HRV) signals. This approach is based on subspaces decomposition of $H R V$ signals using multiscale wavelet packet (MSWP) transform and entropy features extracted from decomposed HRV signals. The detection performance was analyzed using Fisher ranking method, generalized discriminant analysis (GDA) and binary classifier as extreme learning machine (ELM). The ranking strategies designate rank to the available features extracted by entropy methods from decomposed heart rate variability (HRV) signals and organize them according to their clinical importance. The GDA diminishes the dimension of ranked features. In addition, it can enhance the classification accuracy by picking the best discerning of ranked features. The main advantage of ELM is that the hidden layer does not require tuning and it also has a fast rate of detection.

Methodology. For the detection of CAD patients, the HRV data of healthy normal sinus rhythm (NSR) and CAD patients were obtained from a standard database. Self recorded data as normal sinus rhythm (Self_NSR) of healthy subjects were also used in this work. Initially, the HRV time-series was decomposed to 4 levels using MSWP transform. Sixty two features were extracted from decomposed HRV signals by non-linear methods for HRV analysis, fuzzy entropy (FZE) and Kraskov nearest neighbour entropy (K-NNE). Out of sixty-two features, 31 entropy features were extracted by FZE and 31 entropy features were extracted by K-NNE method. These features were selected since every feature has a different physical premise and in this manner concentrates and uses HRV signals information in an assorted technique. Out of 62 features, top ten features were selected, ranked by a ranking method called as Fisher score. The top ten features were applied to the proposed model, GDA with Gaussian or RBF kernal + ELM having hidden node as sigmoid or multiquadric. The GDA method transforms top ten features to only one feature and ELM has been used for classification.

Results. Numerical experimentations were performed on the combination of datasets as NSR-CAD and Self_NSR-CAD subjects. The proposed approach has shown better performance using top ten ranked entropy features. The GDA with RBF kernel + ELM having hidden node as multiquadric method and GDA with Gaussian kernel + ELM having hidden node as sigmoid or multiquadric method achieved an approximate detection accuracy of $100 \%$ compared to ELM and linear discriminant analysis (LDA)+ELM for both datasets. The subspaces level-4 and level-3 decomposition of HRV signals by MSWP transform can be used for detection and analysis of CAD patients.
\end{abstract}

Keywords: multiscale wavelet packet (MSWP) transform; fuzzy entropy (FZE); Kraskov nearest neighbour entropy (K-NNE), generalized discriminant analysis (GDA); extreme learning machine (ELM)

Manuscript received: 02.09.2017

Received in revised form: 07.11.2017

Accepted: 11.11.2017

Address for correspondence: ramsewaknitj@gmail.com 


\section{Introduction}

As per the World Health Organization (WHO) reports, cardiovascular heart diseases are major cause of mortality in the total population. WHO evaluated that $30 \%$ of world population deaths result from cardiovascular heart diseases and expected 23.6 million will endure to these diseases by 2030 [1]. Death rate due to coronary artery disease (CAD) is higher than some other kind of cardiovascular coronary diseases [2]. Coronary artery disease $(\mathrm{CAD})$ is portrayed by narrowing or blockage of coronary artery for the most part caused by atherosclerosis. Atherosclerosis involves plaques and cholesterol accumulates inside the coronary arteries, which impeded the flow of blood into the heart muscles [3]. Because of this, heart pumping is disturbed and may eventually prompt sporadic heartbeat pulses and hence sudden heart failure. The CAD starts in early life and increases gradually. On the off chance that it is not analyzed and treated properly, it will in the end prompt either auricular ischemia or ventricular ischemia or then myocardial infarction. In this way, the starting stage detection of CAD is of prime noticeable quality, and requires an efficient detection approach and heart rate variability (HRV) analysis techniques.

The HRV investigation has turned into a prominent non-invasive diagnostic tool in cardiology to evaluate the activities of the autonomic nervous system (ANS). The HRV is determined by computing time intervals between successive $\mathrm{R}$ peaks points on the QRS complex of the electrocardiogram (ECG) [4]. Morphological format impression of ECG signal is basic yet not sufficient to recognize the presence of CAD because subtle variations are challenging to identify by ECG alone in CAD patients, hence, it is a must to portray these ECG signals into HRV signals $[5,6]$. Over the years, various methods, like time domain, spectral domain and non-linear domain methods have been proposed for examining the variations of these interval values, the purpose of most being the credentials of HRV dissimilarities between ECGs of healthy young, elderly subjects and $\mathrm{CAD}$, cardiac heart failure patients in addition to the study of the autonomic nervous system (ANS), leading to possible prognostic or diagnostic information $[7,8]$. In recent years, a number of investigators have analyzed the HRV by different advanced digital signal processing methods, statistical methods [9], box plot and mean and standard deviation and prominence has been given on classification to detect the normal group and diseased group with different classifiers [10-13].

Advanced digital signal processing approaches can be used in computer-aided diagnosis system to detect the CAD patients. It is a cost effective methods to improve the detection speed of cardiac disease. These systems use features derived by linear, as spectral, time [14] and nonlinear [15] signal processing methods to identify disease related information from cardiovascular signals. Nonlinear techniques are more sensitive than linear methods for recognizing and comprehending the abnormalities of HRV [16]. The explanation behind this better affectability and accuracy has been described by Acharya et al. [6], who demonstrated that the heart is a chaotic (non-linear) oscillator under normal cardiovascular activity. Using non-linear features extracted directly or indirectly from HRV signals, an effective reliable computer supported diagnosis systems can be designed which can detect cardiovascular activity with a high level of accuracy as well as positive affectability values.

The machine learning and artificial intelligence methods are extremely powerful tools for binary and multiclass classification and prediction of cardiac disease. Recently, a new learning process for single hidden layer feedforward neural networks (SLFNs) design called extreme learning machine (ELM) strategy has been widely used in many fields like bio-medical signal analysis and large data analysis [17]. The primary advantage of ELM is that the hidden layer of SLFNs does not require tuning and it also has fast rate of convergence [18]. Indeed, for the arbitrarily picked hidden layer biases and input weights, ELM will prime to a least squares elucidation of a system of non- linear signals for the unidentified output weights having the smallest norm property [18]. Nevertheless, the learning speed of ELM can be thousands of times faster than the traditional feedforward network learning algorithms [17].

In this paper, we have proposed a noninvasive efficient approach using generalized discriminant analysis (GDA) having radial basis function (RBF) or Gaussian (Gausn) kernel function + ELM that can automatically detect the CAD patients using HRV signals. Due to the non-linear nature of HRV signals [19], the multiscale wavelet packet (MSWP) transform [20] is employed to decompose the HRV signals into 4-levels sub space signals. The 64 features are extracted from decomposed sub spaces of HRV signals using fuzzy entropy (FZE) and Kraskov nearest neighbour entropy (K-NNE). Not all the features distinguish well between normal and CAD HRV signals. Therefore, we have used features ranking technique such as the Fisher score, which rank the features according to their clinical significance. The top ten ranked features (highest to lowest, which are ranked by Fisher scheme) are fed to the features reduction scheme as GDA or linear discriminant analysis (LDA). Then, the reduced dimension of features is fed to the extreme learning machine (ELM) binary classifier which differentiates between normal and CAD subjects. The sequence of steps involved in the proposed work is shown in Figure 1. The simulated results show that the proposed detection system achieved an approximate testing accuracy of $100 \%$ for both data sets compared to LDA having regularized solution (Regul. Soln) or singularity solution (Sinty Soln) + ELM having sigmoid or multiquadric hidden nodes and ELM having sigmoid or multiquadric hidden nodes.

The following section explains the HRV signal extraction and detection method. The database and preprocessing are described in Section 2. Methodology is explained in Section 3. Results and discussions are described respectively in sections 4 and 5. Finally the paper is concluded in Section 6. 


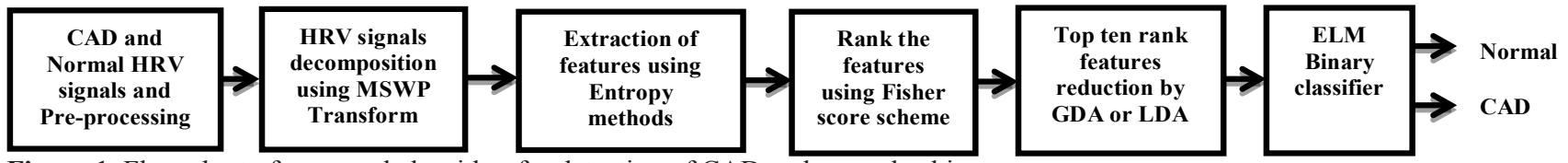

Figure 1. Flow chart of proposed algorithm for detection of CAD and normal subjects.

\section{Materials and pre-processing}

Database

The R-R interval (HRV) data used in this work has been obtained from the ECG signals provided by the normal sinus rhythm (NSR) from MIT BIH database and the CAD data has been obtained from St. Petersburg Institute of Cardiological Technics database [21]. The databases of NSR consisted of eighteen long-term ECG recordings of subjects referred to the Arrhythmia Laboratory at Boston's Beth Israel Hospital. Subjects involved in this database were found to have had no significant arrhythmias; they include five men, aged 26 to 45, and thirteen women, aged 20 to 50 [21]. The database of St. Petersburg Institute of cardiological technics consists of 75 annotated recordings extracted from 32 Holter records. Each record was 30 minutes long and contains standard ECG leads. Only thirteen subjects (9 men and 4 women, aged 1880; mean age: 58) suffered from CAD [21]. The self-recorded data base consists of 13 healthy men subjects, aged between 23 and 32 years. The ECG was continuously recorded for 30 minutes of each subject in the relaxed supine and normal sinus rhythm position in a room free from any kind of disturbance with controlled temperature $\left(22-25^{\circ} \mathrm{C}\right)$. The subjects did not suffer from diabetes and any type of heart disease. The recording of ECG using electrode placement method was done at the sampling rate of $500 \mathrm{~Hz}$ with BIOPAC: MP150 in combination with BIOPAC's "Acqknowledge 4.2" software. The R peaks of the ECG signal were detected using modified Tompkins's algorithm [22,23]. The R-R interval for each subject was then computed. All ECG measurements were taken at the National Institute of Technology, Jalandhar, India.

\section{Pre-processing}

Pre-processing of R-R interval time series data was required before analysis of HRV signals to reduce error and enhances the sensitivity of time series data. First we have done ectopic beat or interval detection and correction before HRV analysis. In this paper, the ectopic beats were detected on the premise of standard deviation filter method which marks outliers as being intervals that lie outside the overall mean R-R interval by a user defined value of standard deviation. The user defined value was used as 3 times the standard deviation [24]. A cubic spline interpolation method was used to replace ectopic intervals located during the detection process. After replacing R-R intervals, they were coded as normal to normal intervals (NN intervals). The NN intervals were sampled at $4 \mathrm{~Hz}$.

Initially, we have selected 2,150 samples of $\mathrm{NN}$ interval for each subject of the database, the first 100 samples and last 50 samples have been excluded from each subject of epoch so that all the subjects were alleviated to the recording atmosphere. This was done in order to provide the prospect of subject evaluation under similar activity levels. To increase in training and testing data size, the remaining 2000 samples of $\mathrm{NN}$ interval were divided into segments of $500 \mathrm{NN}$ intervals. Finally, the segments of NN intervals were used for the feature extraction by MSWP transform.

\section{Methodology \\ HRV signals decomposition by MSWP transform \\ The multiscale Wavelet Packet (MSWP)} transform was first described by Coifman et al. [25]. They comprehensive the link between wavelets and multiresolution approximations. The MSWP may be considered as a tree of sub-spaces, with $\lambda_{0,0}$ denoting the original signal space, i.e., the root node of the tree. In a general notation, the node $\lambda_{l, m}$, with $l$ denoting the scale and $\mathrm{m}$ denoting the subband index within the scale, is decomposed into two orthogonal subspaces as an approximation space $\lambda_{l, \mathrm{~m}} \rightarrow \lambda_{l+1,2 \mathrm{~m}}$ and detail $\lambda_{l, \mathrm{~m}} \rightarrow \lambda_{l+1,2 \mathrm{~m}+1}$ space. This is done by dividing the orthogonal basis $\left\{\theta_{l}\left(\mathrm{t}-2^{l} \mathrm{~m}\right)\right\} \mathrm{m} \varepsilon \mathrm{Z}$ of $\lambda 1$, minto two new orthogonal base $\left\{\theta_{l+1}\left(\mathrm{t}-2^{l+1 \mathrm{~m}}\right)\right\}_{\mathrm{m \varepsilon Z}}$ of $\lambda_{l+1,2 \mathrm{~m}}$ and $\left\{\varphi_{l+1}\left(\mathrm{t}-2^{l+1 \mathrm{~m}}\right)\right\}_{\mathrm{m \varepsilon Z}}$ of $\lambda_{l+1,2 \mathrm{~m}+1}$ [26]. Where $\theta_{l \mathrm{~m}}(\mathrm{t})$ and $\varphi_{l \mathrm{~m}}(\mathrm{t})$ are scaling and wavelet function, respectively and are defined as $\theta_{l \mathrm{~m}}(\mathrm{t})=1 / \sqrt{ }\left|2^{l}\right|\left\{\theta\left(\left(\mathrm{t}-2^{l} \mathrm{~m}\right) / 2^{l}\right)\right\}$ and $\left.\varphi_{l, \mathrm{~m}}(\mathrm{t})=1 / \sqrt{ }\left|2^{l}\right|\left\{\varphi\left(\left(\mathrm{t}-2^{l} \mathrm{~m}\right)\right) / 2^{l}\right)\right\}$, where $2^{l}$ is a scalling parameter and $2^{l} \mathrm{~m}$ is translation function.

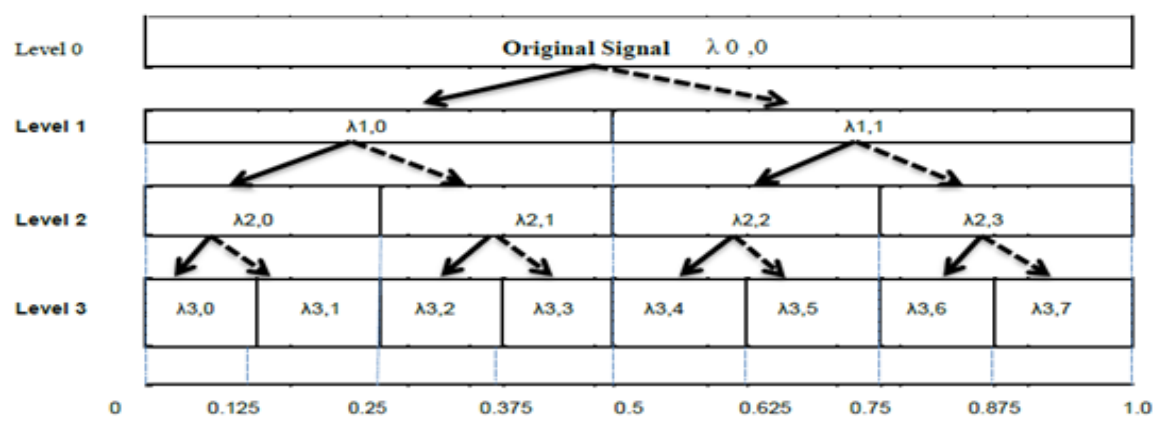

Figure 2. Example of decomposition of MSWP for 3 levels, vertical axis and horizontal axis shows level of decomposition and frequency variety as a fraction of the Nyquist frequency. The $\lambda 1,0, \lambda 1,1 \ldots \ldots \ldots . \lambda 1,1$ represents detail and approximation components of HRV signals. 
The HRV data was decomposed by the MSWP using the Haar wavelet whose mother wavelet function is simply a step function. The dilation and translating for the MSWP were built on powers of 2 or dyadic chunks, e.g., $2^{0}, 2^{1}$, $2^{2}$ etc. The dilation function was repeatedly represented as a tree of high and low pass filters. The first level of the tree decomposes the HRV original data into detail (high frequency, indicated by bold line in Figure 2) and approximation (low frequency, indicated by dotted line in Figure 2) components. Both branches of the tree were split into finer components as $\lambda 0,0$ into $\lambda 1,0$ and $\lambda 1,1$. Figure 2 shows the tree for MSWP for 4 levels of decomposition. A complete description of the method is explained in [20]. The MSWP generated $2^{\text {level }}$ features at each level of decomposition. Hence, the total no of features are 31 for the 4 level decomposition of HRV signals. For the analysis of MSWP transform, the number of samples of HRV per every window and the increments during windows were chosen as 32 and 4, respectively.

\section{Non-linear feature as fuzzy entropy}

Nonlinear features are broadly used to investigate the HRV signals $[10,27]$.These features have ability to extract the concealed nonlinear nature of HRV signals [6]. For finite HRV time series $X=\left\{X_{1}, X_{2} \ldots \ldots \ldots \ldots . X_{M}\right\}$, of length $\mathrm{M}$. The fuzzy entropy (FZE) can be calculated as

$\operatorname{FZE}(\mathrm{X}, \mathrm{m}, \mathrm{N}, \mathrm{R})=\ln \left(\rho^{\mathrm{m}}\right)-\ln \left(\rho^{\mathrm{m}+1}\right)$

Where $\rho^{\mathrm{m}}$ is defined as:

$\rho^{m}(X, N, R)=\frac{1}{M-m} \sum_{i_{1}=1}^{M-m} \frac{1}{M-m-1} \sum_{i_{2}=1, i_{1} \neq i_{2}}^{M-m} e^{-} \frac{\left(D_{i_{1}, i_{2}}\right)^{N}}{R}$.

Again, $\rho^{\mathrm{m}+1}$ calculated same manner for embedding dimension $m+1$. The value of similarity degree $D_{i 1, i 2}$ is calculated through fuzzy function as

$e^{-\frac{\left(D_{i_{1, i_{2}}}\right)^{N}}{R}}$

for HRV time series $\mathrm{X}$, where $\mathrm{N}$ is Fuzzy power and $\mathrm{R}$ is tolerance [28]. In this work, the embedding dimension $\mathrm{m}$, FZE of power N, and tolerance R for all of the data of decomposed HRV were respectively chosen as 4,2 , and 0.15 times the standard deviation of decomposed HRV signals.

\section{entropy}

Non-linear features as Kraskov nearest neighbour

The Kraskov nearest neighbour entropy (K-NNE) estimator KNNE ( X ) of differential entropy for a HRV variable signal $X$ of length $M$ can be calculated as $[29,30]$

$\operatorname{KNNE}(X)=\vartheta(M)-\vartheta(k)+\log \left(c_{D}\right)+D / M \sum_{l=1}^{M} \log [\varepsilon(I)]$

Where $d$ represents the dimension of signal $X$, the $\varepsilon(l)$ is the distance between the $l^{\text {th }}$ sample of HRV signal $\mathrm{X}$ and its $\mathrm{k}$ nearest neighbor. The $\vartheta(\mathrm{k})$ denotes a digamma function and calculated as [29]

$$
\boldsymbol{\vartheta}(\boldsymbol{Y})=1 / \Gamma(\mathrm{Y}) \frac{d \Gamma(Y)}{d Y},
$$

The $\mathrm{c}_{\mathrm{D}}$ denotes a size of $\mathrm{D}$ dimensional unit ball. It is calculated for Euclidean norm [30]

$c_{D}=\frac{\pi^{D}}{\Gamma(1+D / 2)}$
For simulation of K-NNE , k-nearest neighbours sample was chosen as 5 .

\section{Box Plot}

A box plot is a graphical method for representing groups of numerical data along with their quartiles values. It may also have straight lines outspreading vertically from the boxes (whiskers) indicative of erraticism separate the upper and lower quartiles with box plot [46]. Outliers are plotted as separate points or plus symbols. Box plots are non-parametric, they show variety in tests of a measurable data without making any presumptions of the underlying statistical dissemination [47].The dividing between the assorted parts of the case demonstrates the level of scattering (spread) and skewness in the information, and show outliers. Besides, the box plot allows the visual assessment of several parameters, notably the interquartile range, mid-range and median. The labeling of a box plot is shown in Figure 3.

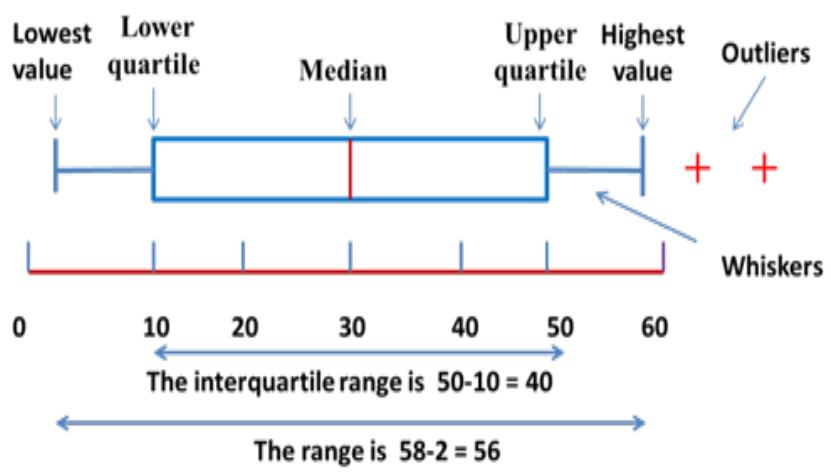

Figure 3. A brief descriptions with proper labeling of box plot.

\section{Features Ranking Method}

Features can be chosen using a feature ranking approach. The ranking approaches allocate ranks to the considered large number of features and array them according to their statistical significance. Further, the lower ranked features can be ignored and higher ranked features can be considered for classification $[31,32]$. These approaches reduce the intricacy of the features dimensions and significantly decreases the time for processing the data without affecting the binary classification enactment. In this work, we have used Fisher score method to rank the features extracted by entropy methods. It is based on filter methods, to rank the features as a pre-processing stride previous to the learning algorithm, and to choose those features having high ranking Fisher score [33].

\section{Generalized discriminant analysis}

As a result of the large changes in the HRV time series patterns of a number of cardiac disease classes, there is commonly a significant similarity between some of the cardiac disease classes like NSR and CAD subjects in the non-linear feature space. In these classes having the larger similarity with each other, it is a challenge to differentiate between the two by ranking methods [34]. In this condition, a feature dimension transformation technique like GDA will 
be very useful. The GDA is a non-linear extension of LDA.

In GDA, the given training data sample is mapped by a kernel function like RBF or Gaussian (Gausn) to a high-dimensional feature space of HRV, where dissimilar classes label of features are made-up to be linearly distinguishable. The LDA technique is then applied to the high-dimensional feature space, where it finds for those vectors that best differentiate among the classes label somewhat than those vectors that best define the training data [35]. Indeed, the aim of the LDA is to search for a transformation matrix that maximizes the ratio of the between-class label scatter to the within-class scatter. In addition, it provides a number of independent features spaces which to be the data [9]. If there are $\beta$ classes label in the given features, the dimension of feature space of HRV can be reduced to $\beta-1$ by GDA method. In this paper, 2 numbers of classes (i.e. binary classes) are taken and the top 10 features are reduced to one feature by GDA. The mathematical expressions of GDA are explained in [35].

\section{Extreme Learning Machine as binary classifier}

Assume that $\left\{\mathrm{X}_{\mathrm{k}}, \mathrm{T}_{\mathrm{k}}\right\}_{\mathrm{k}=1,2 \ldots . \mathrm{m}}$ is a data set of training samples, where for the input features $X_{k}=\left\{X_{k 1}\right.$, $\left.\mathrm{X}_{\mathrm{k} 2}, \ldots \ldots, \mathrm{X}_{\mathrm{Kn}}\right\}^{\mathrm{t}} \mathrm{cR}^{\mathrm{n}}$ are applied to the input nodes and its corresponding target vector value (class label) $\mathrm{T}_{\mathrm{k}} \varepsilon \mathrm{R}$ or $\varepsilon\{-$ $1,1\}$, where $t$ denotes the transpose of the vector matrix. For one of the learning machine method SLFNs, ELM arbitrarily assigned value of weights vector $\mathrm{a}_{\mathrm{s}}=\left\{\mathrm{a}_{\mathrm{s} 1}, \mathrm{a}_{\mathrm{s} 2}, \ldots, \mathrm{a}_{\mathrm{sn}}\right\}^{\mathrm{t}}$ and the bias $\mathrm{B}_{\mathrm{S}} \varepsilon \mathrm{R}$ containing the input layer to $\mathrm{s}^{\text {th }}$ hidden node. The weight vector a_s connecting between input layers to the hidden nodes. The SLFNs with $\mathrm{L}$ number of hidden nodes approximate the input features with zero error if there exists analytically determines the output weight vector $\mathrm{W}=\left\{\mathrm{W}_{1}, \mathrm{~W}_{2}, \ldots \ldots \ldots \mathrm{W}_{\mathrm{L}}\right\}^{\mathrm{t}} \mathrm{c}^{\mathrm{L}}$ connecting between hidden nodes and output nodes [36]. The target value of SLFNs or ELM is formulated as

\section{$\boldsymbol{T}_{k}=\sum_{s=1}^{L} \boldsymbol{W}_{S} \Theta\left(a_{s}, B_{s}, X_{k}\right)$}

for $\mathrm{k}=1,2$, ----, $\mathrm{m}$. Where $\Theta()=.\Theta\left(\mathrm{a}_{\mathrm{s}}, \mathrm{B}_{\mathrm{s}}, \mathrm{X}_{\mathrm{k}}\right)$ is activation function, which represents the output of the sth hidden nodes for the input features samples and parameters. The target vector (generated from output nodes) is linearly related with $\mathrm{W}$ and $\Theta$ hence, it can be formulated in the matrix form as

$\mathrm{H} \mathrm{W}=\mathrm{T}$

Where

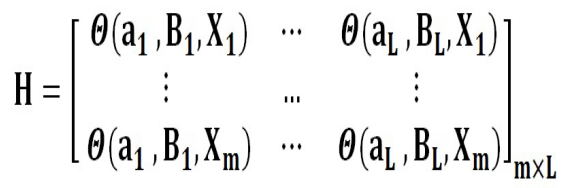

is the output matrix of hidden layer neural network and $\mathrm{T}=\left\{\mathrm{T}_{1}, \mathrm{~T}_{2}, \ldots \ldots, \mathrm{T}_{\mathrm{m}}\right\}^{\mathrm{t}} \varepsilon \mathrm{R}^{\mathrm{m}}$ is the vector of target. To obtain a minimum norm least square solution of $\mathrm{W}$ for the linear system of (13), this can be explicitly achieved to be [18]. $\mathrm{W}=\mathrm{H}^{\dagger} \mathrm{T}$. Where $\mathrm{H}^{\dagger}$ is the inverse of $\mathrm{H}$ matrix, this is known as name of Moore-Penrose generalized inverse [37]. Finally, by accomplishing the solution of $W=\left\{W_{1}\right.$, $\left.\mathrm{W}_{2}, \ldots \ldots \ldots \mathrm{W}_{\mathrm{L}}\right\}^{\mathrm{t}} \varepsilon \mathrm{R}^{\mathrm{L}}$, a decision function $\mathrm{F}($.)is determined for any input of features sample $X=\left\{X_{k 1}, X_{k 2}, \ldots, X_{\mathrm{Kn}}\right\}^{t}$ $\varepsilon \mathrm{R}^{\wedge} \mathrm{n}$. Mathematical expression of $\mathrm{F}($.) is represented as

$$
F(X)=\left(\Theta\left(a_{1}, B_{1}, X\right), \ldots, \Theta\left(a_{L}, B_{L}, X\right)\right) W
$$

Though, intended for binary classification problem, the decision function is based on signum function, and defined as

$$
\mathrm{F}(\mathrm{X})=\operatorname{sign}\left\{\left(\Theta\left(\mathrm{a}_{1}, \mathrm{~B}_{1}, \mathrm{X}\right), \ldots, \Theta\left(\mathrm{a}_{\mathrm{L}}, \mathrm{B}_{\mathrm{L}}, \mathrm{X}\right)\right) \mathrm{W}\right\}
$$

It is important to note that, once the values of the weights vector $\mathrm{a}_{\mathrm{s}} \varepsilon \mathrm{R}^{\mathrm{n}}$ and the bias $\mathrm{B}_{\mathrm{s}} \varepsilon \mathrm{R}$ are arbitrarily assigned at the establishment of the learning algorithm, these values remain fixed and so the elements of matrix $H$ remain constant.

The performance of the proposed method ELM has been tested on additive hidden nodes and RBF. For this test, the activation function $\Theta\left(a_{s}, B_{s}, X\right)$ was considered as the sigmoid function and defined as $\Theta\left(a_{s}, B, X\right)=1 /\left(1+\exp \left(-\left(a^{t} X+B\right)\right)\right.$. For additive nodes, multiquadric function described by [38] as

$\boldsymbol{\Theta}\left(\mathbf{a}, \mathbf{B}_{s}, \mathbf{x}\right)=\sqrt{\left(\|x-\boldsymbol{\alpha}\|_{2}^{2}+\boldsymbol{B}^{2}\right.}$

the function is intended for RBF hidden nodes. Intended for multiquadric and sigmoid activation functions, the hidden node parameters were picked arbitrarily with uniform distribution in $[-0.4,0.4]$. The input weights of the hidden nodes and biases of hidden layer were selected arbitrarily at the starting of the learning for ELM and these values remains constant in every test of simulation. The number of hidden nodes parameter $\mathrm{L}$ was calculated by executing 10 -fold cross-validation on the features with corresponding binary class label. In order to validate our proposed scheme, we have generated arbitrarily pre-defined subjects for training and rest subjects for testing of every data set. These processes were conducted in 20 independent trials and average of testing accuracy was calculated. The accuracy was calculated by using binary classification Gold standard scheme and defined as

$$
\text { Accuracy }=\frac{T P+T N}{T P+T N+F P+F N} \times 100
$$

Where TP, TN, FP, and FN denote number of true positives, true negatives, false positives and false negatives respectively.

\section{Results}

Table I depicts the comparative investigation of NSRCAD dataset and Self_NSR-CAD dataset by top ten ranked features ofFZE and K-NNE in the form of mean and its standard deviation ( $\mathrm{S} \mathrm{d}$.), with their $\mathrm{p}$ value obtained from Wilcoxon rank sum test. Top ten ranked features were extracted by FZE and K-NNE entropy from 4-level decomposition of HRV signals by MSWP transform for both datasets. These ranked features are arranged in the descending order according to their Fisher score, shown in the Table I (top to bottom, 1st and 5 th column) for both datasets. In case of NSR-CAD data set, the features of FZE at decomposition level-4 of HRV signals, approximate (App) and detail (Det) coefficient having higher features score than K-NNE features at decomposition level-4 of HRV signals. While, in case of Self NSR-CAD data set the features of K-NNE at decomposition level-4 at 
first rank then after FZE at decomposition level-4, level-3. Results of Table I also shows that the features extracted by FZE and K-NNE at 4th level and 3rd level of decomposition as App and Det coefficient has lowest p-value $(<0.00001)$. A lower most $\mathrm{p}$-value specifies that this feature has highest discernment ability. Therefore, we can choose 4 th or $3 \mathrm{rd}$ level of decomposition to analyze the NSR subjects and CAD patients. The mean value of FZE features at 4th and 3rd level for CAD patient is higher than NSR and Self_NSR subjects, while, the mean value of K-NNE features at this level of decomposition for NSR is higher than CAD.

Boxplots for the top ten features of FZE and K-NNE entropy estimator are illustrated in Figure 4 for the NSR-CAD dataset. As seen the shapes of box plot related to the NSR and CAD subjects are well separated from the other classes within the same features. In each case, the median values, lower quartile, upper quartile and highest values of features for $\mathrm{CAD}$ subjects are higher than those of NSR subjects. However, lowest values are similar for all top ten features. In the case of NSR subjects, the outliers (indicates the dynamicity of HRV signal) are more compared to CAD subjects hence, these statistical parameters can be differentiated between NSR and CAD subjects.

A graph of number of features versus testing accuracies for several classification methods is shown in Figure 5 (a) and (b) for NSR-CAD and Self_NSR-CAD data set. In this figure, the ELM with several dimension reduction schemes having different kernel functions were used in this work to achieve maximum accuracy of binary classification using the least number of features from the top ten features. Top ten features extracted by FZE and K-NNE from decomposed HRV are organized in the order according to their ranking by Fisher score. These ranked features were fed to the binary classifier one by one up until the highest detection accuracy was reached. In case of the NSR-CAD data set, the proposed classifier achieved approximately $100 \%$ testing accuracy for two features, depicted in Figure 5 (a) While, in case of Self NSR-CAD, the maximum accuracy of $100 \%$ is attained by ELM with multiquadric and sigmoid hidden node+ GDA with Gaussian kernel function for every top ten features, shown in Figure 5 (b). Their results were compared from LDA having Regul. Soln or Sinty Soln + ELM having sigmoid or multiquadric hidden nodes and ELM having sigmoid or multiquadric hidden nodes. This detection was an upsurge in the accuracy with GDA compared to without GDA, a clear result since GDA was used as a feature vector dimension transformation approach and transform to one feature vector by selecting best discriminating of top ten ranked features.

Table I. The Mean and standard deviation ( $\mathrm{S}$ d.) and corresponding $\mathrm{p}$-Value of the top ten ranked features extracted by FZE and K-NNE from 4-level decomposition of HRV signals by MSWP transform for NSR-CAD and Self_NSR-CAD data set. Top ten ranked features arrange on the basis of highest Fisher score to low score (top to bottom, $1^{\text {st }}$ and $5^{\text {th }}$ column) in the table for both data set. Lindicates the level of decomposition; App. represents approximate coefficient and Det. represents detail coefficient of decomposed subspace signals. For statistical significance, if $\mathrm{p}>0.05$ : not significant, $\mathrm{p} \leq 0.05$ : significant and $\mathrm{p}<0.01$ : very significant.

\begin{tabular}{llllllll}
\hline $\begin{array}{l}\text { Features-Level/Approximate } \\
\text { or Detail coefficient }\end{array}$ & $\begin{array}{l}\text { NSR (Mean } \pm \\
\text { S d.) }\end{array}$ & $\begin{array}{l}\text { CAD } \\
(\mathbf{M e a n} \pm \mathbf{S ~ d . )}\end{array}$ & $\mathbf{p - V a l u e}$ & $\begin{array}{l}\text { Features-Level/Approximate } \\
\text { or Detail coefficient }\end{array}$ & $\begin{array}{l}\text { Self-NSR } \\
\text { (Mean } \pm \text { S d.) }\end{array}$ & $\begin{array}{l}\text { CAD (Mean } \pm \\
\text { S d.) }\end{array}$ \\
\hline FZE-L4-App. & $0.038 \pm 0.017$ & $0.12 \pm 0.081$ & $1.30 \mathrm{E}-12$ & K-NNE-L4-App. & $-2.938 \pm 0.666$ & $-1.727 \pm 1$ \\
\hline FZE-L4-Det. & $0.033 \pm 0.016$ & $0.105 \pm 0.071$ & $1.74 \mathrm{E}-12$ & FZE-L4-Det. & $0.029 \pm 0.013$ & $0.101 \pm 0.07$ & $2.71 \mathrm{E}-08$ \\
\hline FZE-L4-Det. & $0.03 \pm 0.015$ & $0.101 \pm 0.07$ & $9.99 \mathrm{E}-10$ & FZE-L3-Det. & $0.021 \pm 0.01$ & $0.08 \pm 0.058$ & $2.33 \mathrm{E}-07$ \\
\hline FZE-L4-App. & $0.039 \pm 0.019$ & $0.115 \pm 0.076$ & $1.05 \mathrm{E}-10$ & FZE-L2-Det. & $0.016 \pm 0.008$ & $0.062 \pm 0.047$ & $2.15 \mathrm{E}-05$ \\
\hline FZE-L4-App. & $0.036 \pm 0.016$ & $0.123 \pm 0.088$ & $2.19 \mathrm{E}-10$ & FZE-L1-App. & $0.011 \pm 0.006$ & $0.048 \pm 0.037$ & $1.7 \mathrm{E}-04$ \\
\hline K-NNE-L4-App. & $-2.931 \pm 0.737$ & $-1.72 \pm 1.004$ & $1.61 \mathrm{E}-10$ & K-NNE-L4-App. & $-2.857 \pm 0.661$ & $-1.72 \pm 1.004$ & $9.6 \mathrm{E}-07$ \\
\hline FZE-L3-App. & $0.025 \pm 0.015$ & $0.082 \pm 0.056$ & $2.23 \mathrm{E}-09$ & FZE-L4-App. & $0.038 \pm 0.021$ & $0.123 \pm 0.088$ & $8.30 \mathrm{E}-07$ \\
\hline FZE-L3-Det. & $0.023 \pm 0.015$ & $0.08 \pm 0.058$ & $1.26 \mathrm{E}-09$ & FZE-L3-App. & $0.028 \pm 0.014$ & $0.082 \pm 0.056$ & $8.23 \mathrm{E}-07$ \\
\hline K-NNE-L4-Det. & $-3.062 \pm 0.891$ & $-1.761 \pm 1.041$ & $3.18 \mathrm{E}-09$ & FZE-L4-Det. & $0.037 \pm 0.018$ & $0.105 \pm 0.071$ & $9.38 \mathrm{E}-06$ \\
\hline K-NNE-L3-App. & $-3.409 \pm 0.891$ & $-2.108 \pm 1.041$ & $3.18 \mathrm{E}-09$ & K-NNE-L3-Det. & $-3.691 \pm 0.733$ & $-2.293 \pm 1.343$ & $5.86 \mathrm{E}-06$ \\
\hline
\end{tabular}
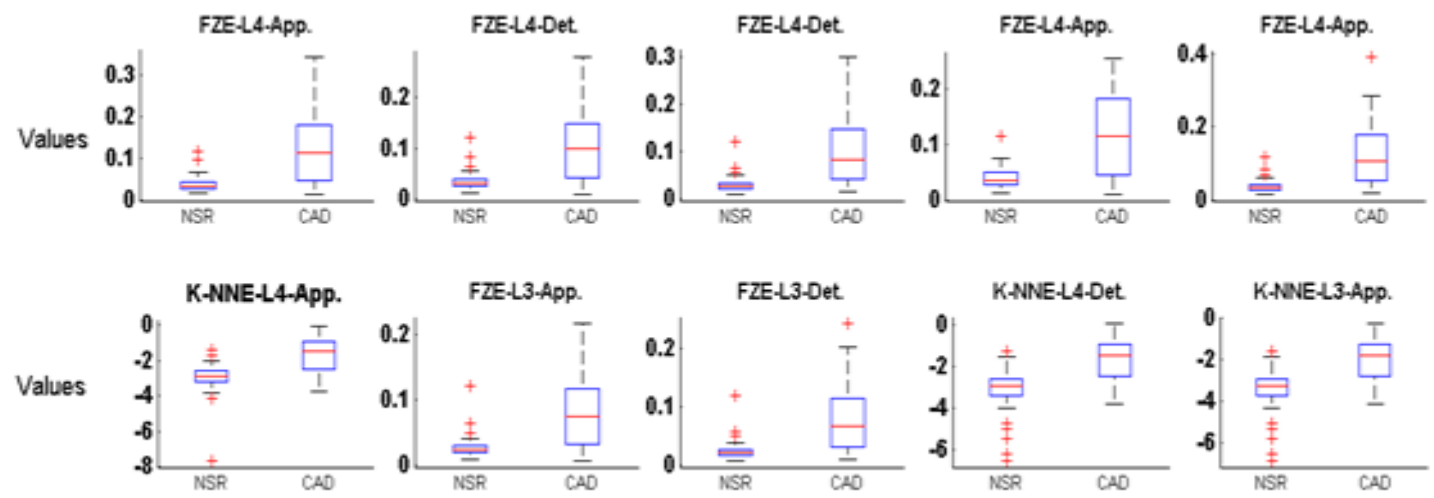

Figure 4. Box plot of top ten ranked features extracted by non-linear FZE and K-NNE method from decomposed HRV signals by MSWP for NSR-CAD data set. 


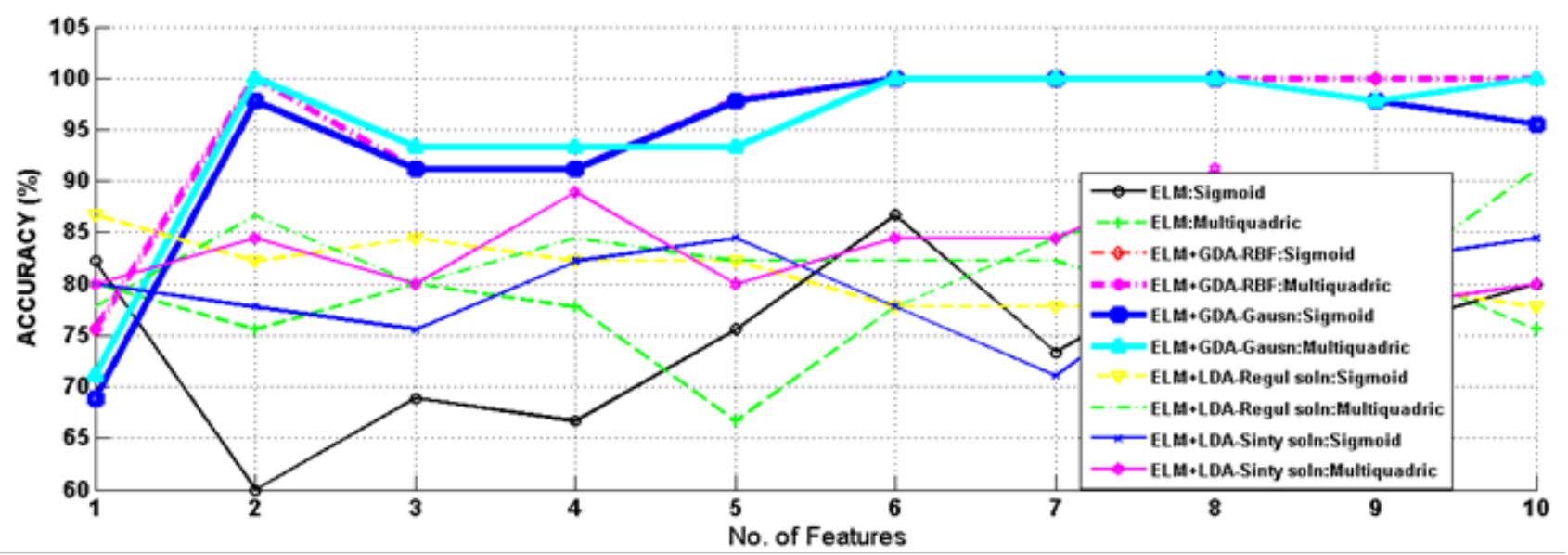

a

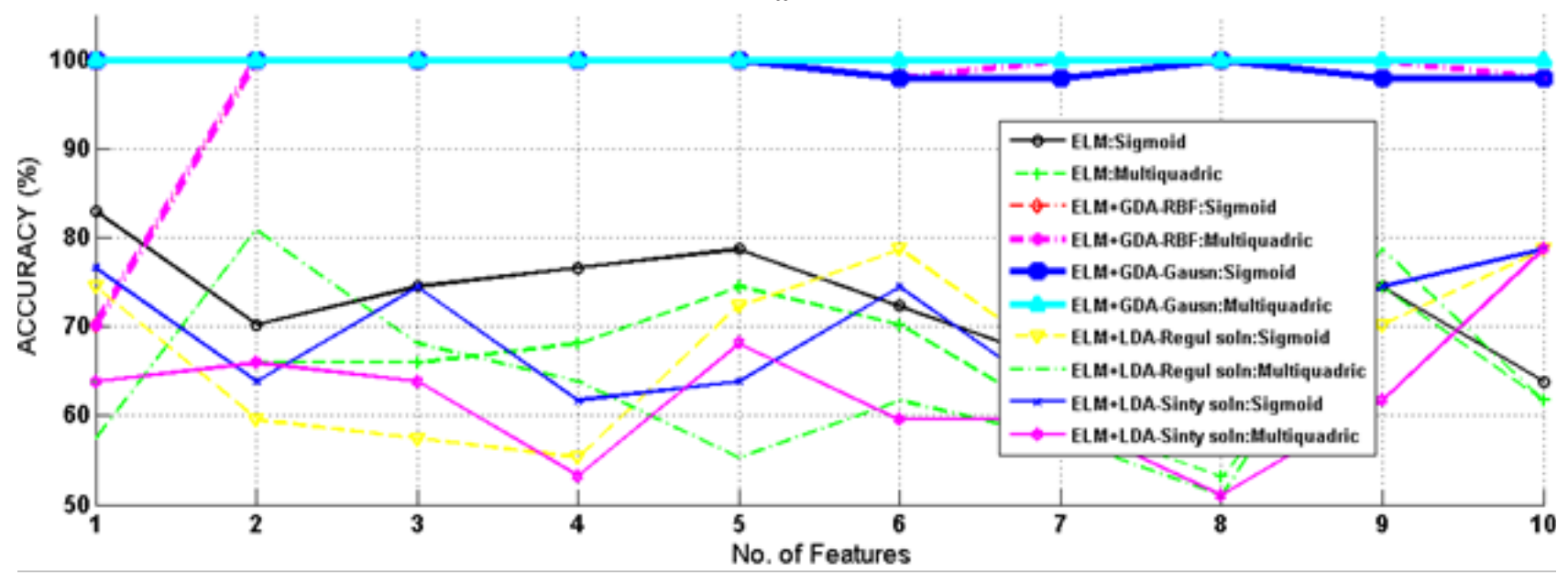

b

Figure 5. Plots depicting detection accuracies for different number of top ten ranked features with GDA + ELM, LDA+ ELM having different kernel functions and hidden nodes and ELM having hidden nodes as sigmoid and multiquadric for (a) NSR-CAD (b) Self_NSR-CAD.

\section{Discussion}

In Table II, an ephemeral summary of investigation carried out by many authors for HRV signals, study the features using several techniques like discrete wavelet transform (DWT), flexible analytic wavelet transform (FAWT), wavelet packet transform (WPT) [32,39], different features dimension reduction approach namely principal component analysis (PCA), independent component analysis (ICA), LDA, GDA [35,40], and several binary classification techniques as k-nearest neighbors (KNN) algorithm, Support vector machine (SVM), artificial neuron network (ANN), ELM exploring in terms of accuracy investigated on CAD patients and healthy subjects. It shows that broad research has been completed in recent years in the study and detection of CAD patients with different methods. Babak et al. [35], Patidar et al. [41], Lee et al. [12] and Mohit et al. [32] have employed HRV study using linear and non-linear methods for features extraction and t-test, PCA, GDA as an features diminishment scheme, while classifying tried for different approaches and found SVM and least squares support vector machine (LS-SVM) as better with $95.77 \%, 90 \%, 99.72 \%$ and $100 \%$ accuracy.

In this paper, the non-linear features extracted by FZE and K-NNE methods from 4 level decomposed HRV using MSWP transform were carried out for the HRV analysis acquired from ECG recording of NSR subjects and CAD patients. The mean value of FZE features at 4 th and 3rd level for CAD patient is higher than NSR subjects since, the variability in NN interval tachogram more in the NSR group than CAD affected patients. While the mean value of K-NNE features at this level of decomposition for NSR patients was higher than for CAD patients. Further, the extracted features by non-linear methods for HRV analysis has been used with Fisher score ranking method and GDA as feature dimension transform technique as classifier modules to obtain an approximate accuracy of $100 \%$ and without GDA an accuracy of $85 \%$. Overall there was an increase in the accuracy with GDA compared to without GDA, an obvious result since Fisher score scheme and GDA were used as top ten features ranked on the basis of Fishers score and feature space transformation technique. 
Table II. An ephemeral summary and assessment of the classification accuracy of the proposed work with the existing work.

\begin{tabular}{|c|c|c|c|c|}
\hline Authors & Methods & Features & Classifier & Accuracy \% \\
\hline Karimi et al. [3940] & DWT, HSS and WPT & Several statistical features & ANN & 90 \\
\hline Lee et al. [12] & $\begin{array}{l}\text { Nonlinear methods, Linear, } \\
\text { and reduced features by t-test }\end{array}$ & $\begin{array}{l}5 \text { nonlinear features and } 6 \\
\text { Linear }\end{array}$ & SVM & 90 \\
\hline Babak et al. [35] & $\begin{array}{l}\text { Linear, non linear methods and features } \\
\text { reduced by GDA and LDA }\end{array}$ & $\begin{array}{l}7 \text { time domain, } 1 \text { frequency } \\
\text { and } 7 \text { non-linear }\end{array}$ & SVM & 95.77 \\
\hline Zhao and Ma [4243] & EMD, and TEO & Several statistical features & BPNN & 85 \\
\hline Babaoglu et al. [4344] & GA, EST and BPSO & 11 Features & SVM & 81.46 \\
\hline Babaoglu et al. [4445] & EST and features reduced by PCA & 18 Features & SVM & 79.17 \\
\hline Dua et al. [4041] & Features reduced by PCA & 6 Nonlinear features & MLP & 89.5 \\
\hline Giri et al. [11] & DWT, features reduced by ICA, & 10 Features & GMM & 96.8 \\
\hline Patidar et al. [4142] & TQWT and features reduced by PCA & 2 Entropy features & $\begin{array}{l}\text { LS-SVM with Morlet } \\
\text { wavelet kernel }\end{array}$ & 99.72 \\
\hline Nan Liu et al. [4546] & Selective and total segments & Linear and frequency feat & ELM \& SVM & $68.48 \& 71.20$ \\
\hline Mohit et al. [32] & $\begin{array}{l}\text { FAWT and ranking method like } \\
\text { Entropy, ROC and Bhattacharya }\end{array}$ & $\begin{array}{l}\text { FzEn and K-NN Entropy } \\
\text { estimator }\end{array}$ & $\begin{array}{l}\text { LS-SVM with RBF \& } \\
\text { Morlet wavelet kernel }\end{array}$ & 100 \\
\hline Monappa et al. [4] & $\begin{array}{l}\text { Linear and non-linear, features reduced } \\
\text { by PCA }\end{array}$ & \begin{tabular}{|l} 
Various time domain, \\
Frequency domain and \\
non-linear domain
\end{tabular} & PNN, KNN and SVM & $\begin{array}{l}\text { Without PCA: } 68.33,76.67 \text { and } \\
90.00 \\
\text { With PCA: } 68.33,85.00 \text { and } 91.67\end{array}$ \\
\hline This work & $\begin{array}{l}\text { MSWP transform, Fisher ranking } \\
\text { method and features reduced by } \\
\text { GDA,LDA }\end{array}$ & $\begin{array}{l}31 \text { features by K-NNE \& } 31 \\
\text { features by FZE method. }\end{array}$ & ELM & 100 \\
\hline
\end{tabular}

Our proposed method has the following novelty:

(a) The proposed method uses only one feature after reduction of top ten features to attain the excellent accuracy of $100 \%$. However, Patidar et al. [41] also achieved classification testing accuracy of $99.72 \%$ by only two features.

(b) Results are robust: due to 10-fold crossvalidation has been used for selection of hidden nodes and extracted features of each subjects generated randomly for training and rest subjects for testing of every data set.

(c) The proposed algorithm can be employed in advanced signal processing system like a computer aided diagnostic system. As, this method only needs one feature for classification and is using ELM for detection of CAD patients, the diagnosis of CAD will be fast. Due to this, in future, online CAD detection system can be possible for clinical applications.

\section{Limitations of our proposed method:}

(a) Before the application of the proposed approach in diagnosing of CAD patients, the algorithm should be trained by large data sets of HRV signals.

\section{Conclusion}

In this work, a new approach has been proposed for the detection of CAD patients based on extracted features dimension reduction technique as GDA with RBF or Gaussian kernel function and binary classifier as ELM having multiquadric RBF hidden nodes. It was concluded based on the detection accuracy graph that the proposed hybrid GDA + ELM coronary artery disease detection algorithm achieved 100\% accuracy. The features extracted by FZE and K-NNE at 4th level and 3rd level of decomposition as approximate and detail coefficient had the lowest p-value
$(<0.00001)$. Therefore, 4th or 3rd level of decomposition of HRV signals by MSWP transform may be used for analysis of CAD patients. The mean value of FZE features at 4th and 3rd level for CAD patient was higher than for NSR subjects, while the mean value of K-NNE features at this level of decomposition for NSR was higher than CAD patients. Hence, our proposed approach scheme could be used as a part of medicinal facilities, polyclinics, health care and group screening to help cardiologists in their standard determination. This approach needs only one feature for binary classification using ELM, after transformation of top ten entropy, therefore the diagnosis of CAD will be fast. Due to this, in future, online cardiac heart diseases detection systems can be possible for clinical purpose.

\section{Acknowledgments}

The authors are extremely obliged to the learned reviewers for their helpful comments that significantly improved this paper.

\section{References}

1. World Health Organization. Global status report on noncommunicable diseases. 2010. Available from: http://www.who. $\mathrm{int} / \mathrm{nmh} /$ publications/ncd_report2010/en/

2. Wong ND. Epidemiological studies of CHD and the evolution of preventive cardiology. Nat Rev Cardiol. 2014;11:276-289.

3. National Heart Lung and Blood Institute, What is coronary heart disease? Available from: http://www.nhlbi.nih.gov/health/ health-topics/topics/cad/

4. Poddar MG, Kumar V, Sharma YP. Automated diagnosis of coronary artery disease patients by heart rate variability analysis using linear and non-linear methods. J Med Eng Technol. 2015;39:331-341.

5. Silber E. Katz LN. Heart Disease. New York: Macmillan Publishing Co; 1975: p 498. 
6. Acharya UR, Kannathal N, Krishnan SM. Comprehensive analysis of cardiac health using heart rate signals. Physiol Meas. 2004;25:1139-1151.

7. Nikolopoulos S, Alexandridi A, Nikolakeas S, Manis G. Experimental analysis of heart rate variability of long-recording electrocardiograms in normal subjects and patients with coronary artery disease and normal left ventricular function. J Biomed Inform. 2003;36:202-217.

8. Manis G. Nikolopoulos S, Alexandridi A. Davos C. Assessment of the classification capability of prediction and approximation methods for HRV analysis. Comput Biol Med. 2007;37:642-654. 9. Martinez AM, Kak AC. PCA versus LDA. IEEE Trans Pattern Anal Mach Intell. 20012;3:228-233.

10. Acharya UR, Faust O, Sree V, Swapna G, Martis RJ, Kadri NA, et al. Linear and nonlinear analysis of normal and CADaffected heart rate signals. Comput Methods Programs Biomed. 2014;113:55-68.

11. Poddar MG, Kumar V, Sharma YP. Linear-nonlinear heart rate variability analysis and SVM based classification of normal and hypertensive subjects. Journal of Electrocardiology. 2013;46:25-30. 12. Giri DG, Acharya UR, Martis RJ, Sree VS, Lim TC, Ahamed TVI, Suri JS. Automated diagnosis of coronary artery disease affected patients using LDA, PCA, ICA and discrete wavelet transform. Knowledge Based Systems, 2013;37:274-282.

13. Lee H, Noh K, Ryu K. Mining biosignal data: Coronary artery disease diagnosis using linear and nonlinear features of HRV. Int T Washio et al. (Eds.) PAKDD 2007 Workshops LNAI 4819 Springer-Verlag Berlin Heidelberg; 2007: p 218-228.

14. Sun Y, Chan K L, Krishnan SM. Arrhythmia detection and recognition in ECG signals using nonlinear techniques. Ann Biomed Eng. 2000; 28:37-40.

15. Pincus SM. Approximate entropy as a measure of system complexity. Proc Nat Acad Sci U S A. 1991;88:2297-2301.

16. Goldberger AL, West BJ. Applications of nonlinear dynamics to clinical cardiology. Ann N Y Acad Sci. 1987;504:195-213.

17. Huang GB, Zhu Q Yu, Siew CK. Extreme learning machine: Theory and applications. Neurocomputing. 2006;70:489-501.

18. Huang GB, Wang DH, Lan Y. Extreme learning machines: a survey. Int J Mach Learn Cybernet. 2011;2:107-122.

19. Bayram I. An analytic wavelet transform with a flexible timefrequency covering. IEEE Transactions on Signal Processing, 2013;61:1131-1142.

20. Khushaba RN, Kodagoda S, Lal S, Dissanayake G. Driver drowsiness classification using fuzzy wavelet-packetbased feature-extraction algorithm. IEEE Trans Biomed Eng. 2011;58:121-131.

21. Goldberger AL, Amaral LAN, Glass L, Hausdorff JM.: PhysioBank, PhysioToolkit, and PhysioNet Components of a New Research Resource for Complex Physiologic Signals. Circulation. 2000; 101: 215- 220. (http://www.physionet.org).

22. Tompkins JW. Bio Medical Signal Processing. Book Phi publisher, 7th edition.

23. Pan J, Tompkins WJ. A real-time QRS detection algorithm. IEEE Trans Biomed Eng. 1985;32:230-236.

24. Mitov IP. A method for assessment and processing of biomedical signals containing trend and periodic components. Med Eng Phys. 1998;20:660-668.

25. Coifman RR, Meyer Y, Quake S, Wickerhauser V. Wavelet analysis and Signal processing. Wavelets and Their Applications, Sudbury, MA: Jones and Barlett. 1992; 2:153-178.

26. Mallat S. A Wavelet Tour of Signal Processing. The Sparse
Way, 3rd ed. New York: Academic; 2009: p 4-7.

27. Rajendra Acharya U, Faust O, Adib Kadri N, Suri JS, Yu W. Automated identification of normal and diabetes heart rate signals using nonlinear measures. Comput Biol Med. 2013;43:1523-1529. 28. Azami H, Fernández A, Escudero J. Refined multiscale fuzzy entropy based on standard deviation for biomedical signal analysis. Med Biol Eng Comput. 2017;55:2037-2052.

29. Kraskov A. Stögbauer H, Grassberger P. Estimating mutual information. Phys Rev E Stat Nonlin Soft Matter Phys. 2004 Jun;69(6 Pt 2):066138.

30. Veselkov KA, Pahomov VI, Lindon JC, Volynkin VS, Crockford D, Osipenko GS, et al. A metabolic entropy approach for measurements of systemic metabolic disruptions in pathophysiological States. J Proteome Res. 2010;9:3537-3544.

31. Duda RO, Hart PE, Stork DG. Pattern classification. John Willey \& Sons; 2000; p 2.

32. Kumar M, Pachori RB, Acharya UR. An efficient automated technique for CAD diagnosis using flexible analytic wavelet transform and entropy features extracted from HRV signals. Expert Systems with Applications. 2016;63:165-172.

33. Quanquan Gu, Zhenhui Li, Jiawei H. Generalized Fisher Score for Feature Selection. Proceedings of the Twenty-Seventh Conference on Uncertainty in Artificial Intelligence, Barcelona, Spain, 2011; July 14-17.

34. Kampouraki A, Manis G, Nikou C. Heartbeat time series classification with support vector machines. IEEE Trans Inf Technol Biomed. 2009; 13: 512-518.

35. Asl BM, Setarehdan SK, Mohebbi M. Support vector machinebased arrhythmia classification using reduced features of heart rate variability signal. Artif Intell Med. 2008;44:51-64.

36. Balasundaram S, Gupta D, Kapil. 1-Norm extreme learning machine for regression and multiclass classification using Newton method. Neurocomputing 2014; 128: 4-14.

37. Rao CR, Mitra SK. Generalized Inverse of Matrices and its Applications. John Wiley: New York; 1971.

38. Huang GB, Zhou H, Ding X, Zhang R. Extreme learning machine for regression and multiclass classification. IEEE Trans Syst Man Cybern B Cybern. 2012,42:513-529.

39. Karimi M, Amirfattahi R, Sadri S, Marvasti SA. Noninvasive detection and classification of coronary artery occlusions using wavelet analysis of heart sounds with neural networks. In the 3rd IEE international seminar on medical applications of signal processing 2005, 117-120.

40. Dua S, Du X, Sree SV. Novel classification of coronary artery disease using heart rate variability analysis. Journal of Mechanics in Medicine and Biology. 2012;12:124-129.

41. Patidar S, Pachori RB, Acharya UR. Automated diagnosis of coronary artery disease using tunable-Q wavelet transform applied on heart rate signals. Knowledge-Based Systems. 2015;82:1-10.

42. Zhao Z. Ma C. An intelligent system for noninvasive diagnosis of coronary artery disease with EMD-TEO and BP Neural Network. In International workshop on education technology and training and international workshop on geoscience and remote sensing 2008, 2: pp 631-635.

43. Babaoglu I, Findik O, Ülker E. A comparison of feature selection models utilizing binary particle swarm optimization and genetic algorithm in determining coronary artery disease using support vector machine. Expert Systems with Applications. 2010;37:3177-3183.

44. Babaoglu I, Findik O, Bayrak M. Effects of principle component analysis on assessment of coronary artery diseases 


\section{Original Research}

using support vector machine. Expert Systems with Applications. 2010;37:2182-2185.

45. Nan L, Zhiping L, Zhixiong K. Patient Outcome Prediction with Heart Rate Variability and Vital Signs. J Sign Process Syst. 2011;64:265-278.
46. Pfannkuch M. Comparing box plot distributions: a teacher's reasoning. Statistics Education Research Journal. 2006;5:27-45.

47. Ramos MF, Tian TS. The shifting boxplot: a boxplot based on essential summary statistics around the mean. International Journal of Psychological Research. 2010;3:37-45. 